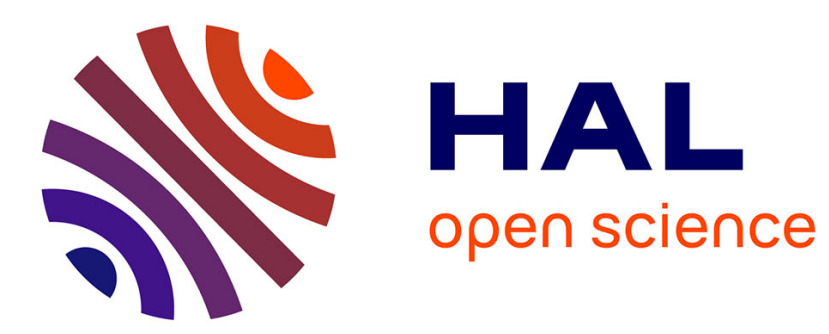

\title{
Adaptation du spectrophotomètre " Cary 14 " à l'étude des spectres et des déclins de luminescence
}

\author{
O. de Witte, Y.H. Meyer
}

\section{To cite this version:}

O. de Witte, Y.H. Meyer. Adaptation du spectrophotomètre "Cary 14 " à l'étude des spectres et des déclins de luminescence. Revue de Physique Appliquée, 1966, 1 (2), pp.109-113. 10.1051/rphysap:0196600102010900 . jpa-00242691

\section{HAL Id: jpa-00242691 https://hal.science/jpa-00242691}

Submitted on 1 Jan 1966

HAL is a multi-disciplinary open access archive for the deposit and dissemination of scientific research documents, whether they are published or not. The documents may come from teaching and research institutions in France or abroad, or from public or private research centers.
L'archive ouverte pluridisciplinaire HAL, est destinée au dépôt et à la diffusion de documents scientifiques de niveau recherche, publiés ou non, émanant des établissements d'enseignement et de recherche français ou étrangers, des laboratoires publics ou privés. 


\title{
ADAPTATION DU SPECTROPHOTOMËTRE « CARY 14 » A L'ÉTUDE DES SPECTRES ET DES DÉCLINS DE LUMINESCENCE
}

\author{
Par O. DE WITTE et Y. H. MEYER, \\ Laboratoire de Physique de l'École Polytechnique.
}

\begin{abstract}
Résumé. - Un " luminographe » a été réalisé, qui permet d'effectuer rapidement par une méthode d'échantillonnage et d'observation différée :

$1^{0}$ L'enregistrement des spectres de fluorescence.

$2^{\circ}$ L'enregistrement direct des spectres de phosphorescence après excitation, l'intervalle de temps étant compris entre $10^{-1}$ et $4 \times 10^{-2}$ seconde.

3o La mesure de la durée du déclin d'une raie de luminescence avec une précision de l'ordre de l'unité pour cent quand cette durée est supérieure à $10^{-4} \mathrm{~s}$. L'isolement de la raie est celui que permet la résolution spectrale du CARY 14.
\end{abstract}

\begin{abstract}
Adaptation of the "Cary 14" spectrophotometer to the study of luminescence spectra and decays.

This "Luminograph" working on the principle of sampling time resolved spectroscopy is useful for :

10 Direct recording of fluorescence spectra during excitation.

$2^{\circ}$ Direct recording of phosphorescence spectra at a time interval of between $10^{-4}$ and $4 \times 10^{-2}$ second after excitation.

$3^{0}$ Measurement of luminescence decays that are longer than $10^{-4}$ second with a precision of one per cent and within the spectral resolution of the Cary 14.
\end{abstract}

L'utilisation du spectrophotomètre CARY 14 pour l'enregistrement des spectres de phosphorescence a déjà été indiquée [1]. Le montage décrit ne permettait pas de mesurer les durées de vie de l'ordre de la milliseconde. Nous avons réalisé à l'aide du même spectrophotomètre enregistreur un " luminographe ", c'est-à-dire un montage permettant d'obtenir en quelques minutes :

10 Le spectre de luminescence totale (" fluorescence " observée pendant l'excitation).

$2^{\circ}$ Le spectre de luminescence de durée de vie supérieure à un temps réglable à volonté (minimum $10^{-4} \mathrm{~s}$ ) (" phosphorescence " observée après l'excitation).

$3^{\circ}$ La valeur de durée de vie (supérieure à $2 \times 10^{-4} \mathrm{~s}$ ) de chaque raie (avec une résolution spectrale de quelques angströms).

Un gain de temps important est donc réalisé dans la manipulation. Ceci est particulièrement avantageux lorsque des recherches systématiques doivent être conduites sur un grand nombre d'échantillons dont la préparation et la mise en place aux conditions de mesure dans un cryostat, à basse température par exemple, constituent la phase la plus longue.

Rappel du fonctionnement du CARY 14. Lorsque le spectrophotomètre enregistreur à double faisceau CARY 14 est utilisé en absorption, le détecteur photoélectrique reçoit alternativement les signaux lumineux traversant la cuve de référence et la cuve échantillon.

Le CARY 14 est également prévu pour fonctionner en simple faisceau. Le signal électrique de référence n'est plus alors fourni par le photomultiplicateur mais est remplacé par une tension continue de référence fixée par le constructeur. On peut ainsi enregistrer le spectre d'émission d'une source continue de lumière entre 2000 et $25000 \AA$ avec une résolution (de 1 à $3 \AA$ ) permise par le système dispersif (prisme plus réseau) si la brillance de la source est suffisante.

L'appareil ne travaillant plus en différentiel, le spectre obtenu est modulée par la sensibilité spectrale des détecteurs. En simple faisceau le photomultiplicateur observe le signal émis par la source pendant une fraction de la période définie par un disque qui hache le faisceau lumineux. Avec une alimentation en 50 hertz, le cycle total dure 40 millisecondes au cours desquelles le photomultiplicateur effectue les quatre mesures suivantes (fig. 1a) :

- pendant les deux intervalles obscurs, réglage du zéro par servomoteurs asservis ;

- pendant l'intervalle de référence, étalonnage des amplificateurs de mesure par servomoteurs asservis à la tension fixe de référence ;

- pendant l'intervalle d'observation, mesure du signal lumineux ayant traversé le monochromateur.

Ces intervalles ont des durées théoriques de $10 \mathrm{~ms}$ qui correspondent à la rotation du disque. En fait, la 
1 cycle $=40$ millisecondes.

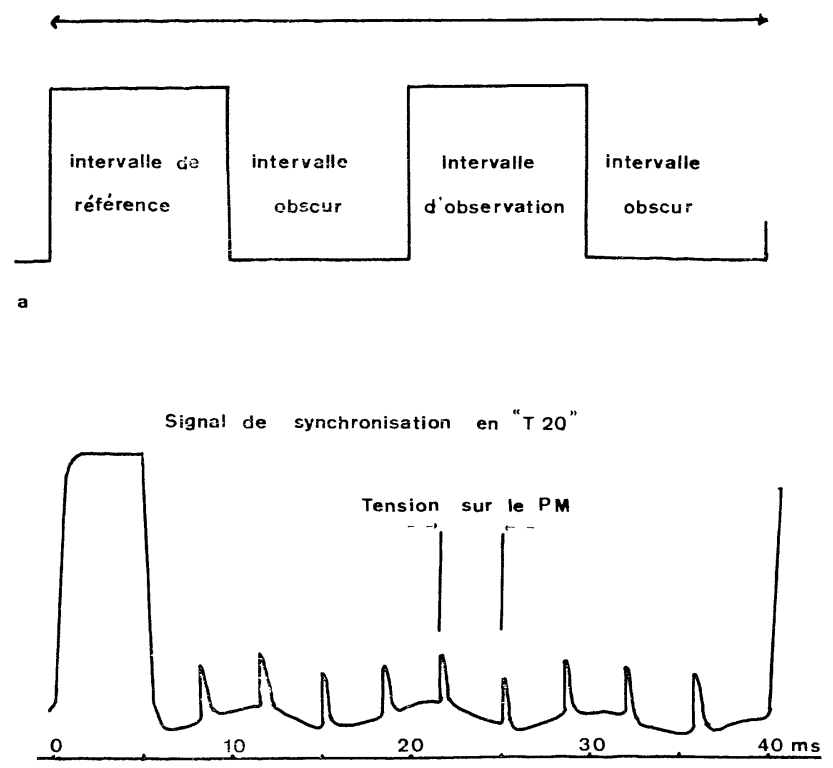

Fig. 1. - a) Cycle de fonctionnement du CARY 14 b) Signal de synchronisation en ( $\mathrm{T} 20$ » observé à l'oscillographe.

haute tension n'est appliquée au photomultiplicateur par des relais que pendant $4 \mathrm{~ms}$.

Pendant les trois autres intervalles, le signal d'observation est gardé en mémoire sur une capacité. Etant synchronisé avec la période du secteur, le CARY 14 délivre des impulsions de référence correspondant aux divers intervalles d'un cycle.

Ces signaux sont repérés sur le CARY 14 en des points facilement accessibles (" $\mathrm{T} 19$ », " $\mathrm{T} 20$ », “ T 21 »). Nous utilisons le signal “ T 20 » (fig. $1 b)$ qui permet de repérer facilement l'intervalle d'observation entre la cinquième et la sixième impulsion intermédiaire et donne une référence de temps précise.

Principe du luminographe (fig. 2). - La substance dont on veut étudier le spectre de photoluminescence est placée dans un tube ou éventuellement dans un cryostat à azote liquide. L'excitation est obtenue à l'aide d'un tube à décharge au xénon, en silice non fluorescente, réalisé par la Société VERRE et QUARTZ et déclenché à chaque cycle de fonctionnement du CARY 14. Le flash alimenté sous $1 \mathrm{kV}$ utilise 2 joules par éclair, soit à $25 \mathrm{~Hz}$ une puissance moyenne de 50 watts. Un filtre cylindrique peut être placé entre le flash et l'échantillon. Selon l'instant de déclenchement du flash d'excitation par rapport à l'intervalle d'observation, nous pourrons avoir les trois modes de fonctionnement ci-dessous :

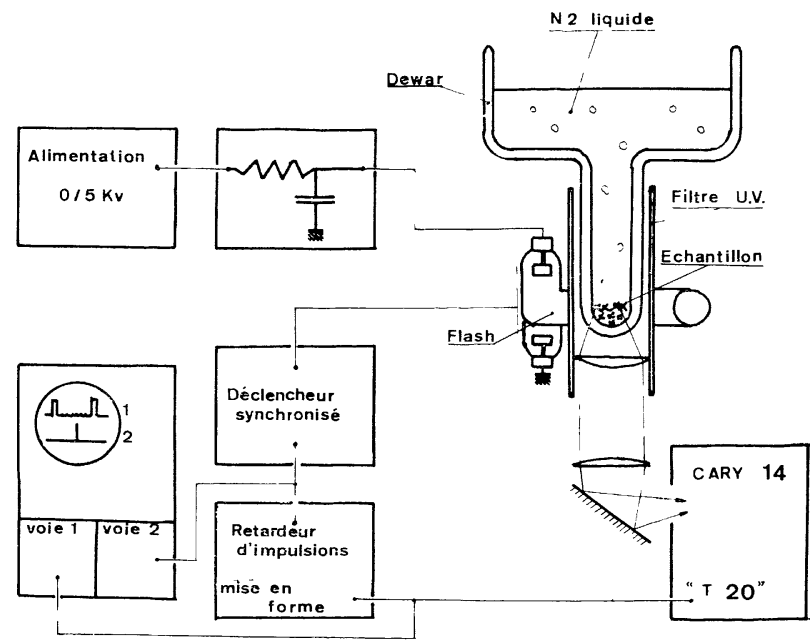

FIG. 2. - Schéma synoptique du luminographe.

1) Spectre de " Luminescence totale ». L'impulsion prise en " $\mathrm{T} 20$ » sur la branche ascendante d'un grand créneau est retardée d'environ $23 \mathrm{~ms}$ et sert ainsi à déclencher le flash d'excitation pendant l'intervalle d'observation. Dans le cas le plus fréquent d'une excitation ultraviolette, la lumière parasite du flash aux longueurs d'onde
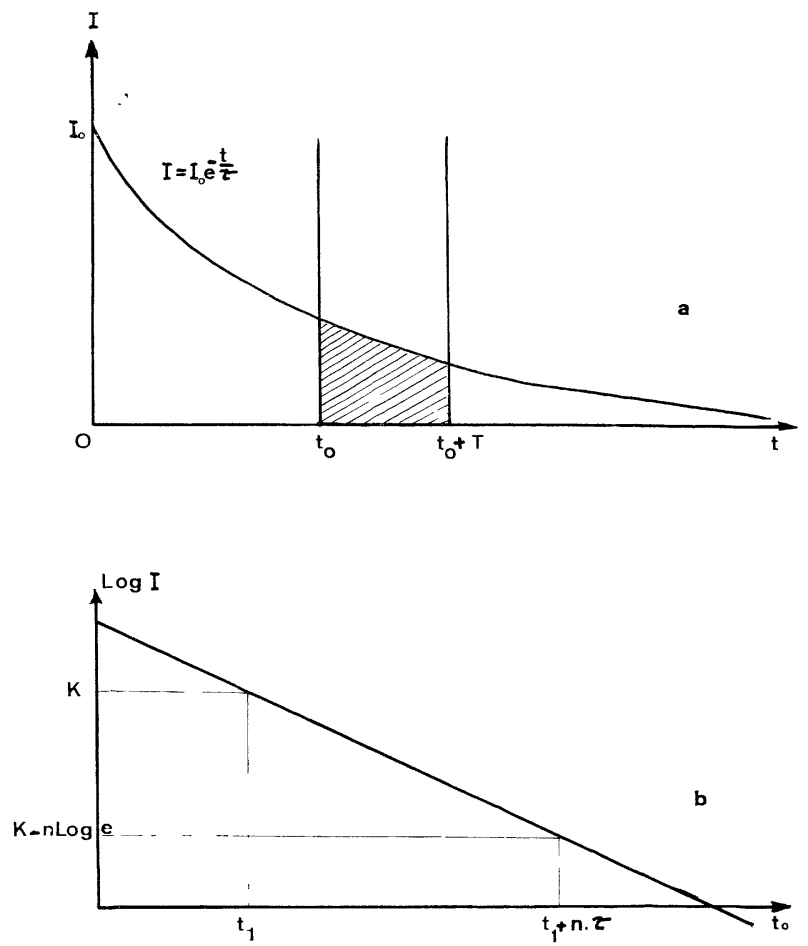

FIg. 3. - - a) Courbe du déclin de la luminescence après chaque éclair. Le photomultiplicateur observe entre $t_{\mathbf{0}}$ et $t_{0}+T$.

b) Courbe du déclin obtenu par échantillonnage en fonction du ( temps d'observation ». 
d'observation est supprimée à l'aide d'un verre de Wood.

L'utilisation d'un tube à éclairs périodiques de grande puissance instantanée permet une augmentation du rapport signal sur bruit, une bonne utilisation de l'énergie d'excitation et par suite, une réduction considérable de l'échauffement du montage et de l'échantillon.

2) Spectres de “ Phosphorescence) (durée de vie supérieure à $10^{-4} \mathrm{~s}$ ). - L'impulsion en « T 20 ») est moins retardée que précédemment. On peut ainsi observer les spectres de phosphorescence un " temps d'observation " $t_{0}$ après l'excitation. En faisant varier $t_{0}$, on peut enregistrer des spectres de phosphorescence entre 0 et $36 \mathrm{~ms}$ après l'excitation. Une résolution dans le temps de plusieurs spectres de phosphorescence est ainsi possible.

3) Mesure des déclins exponentiels. - Le système dispersif étant calé au maximum d'une raie d'émission il suffit alors de faire varier le temps d'observation et de noter l'intensité lumineuse correspondante.

En effet ( $f g .3 a$ ), lorsque le photomultiplicateur observe un signal à décroissance exponentielle $I=I_{0} \mathrm{e}^{-t / \mathrm{v}}$ entre les instants $t_{0}$ après le début du signal et $t_{0}+T$ l'intensité moyenne enregistrée est proportionnelle à :

$$
\int_{t_{0}}^{t_{0}+T} I \mathrm{~d} t \text { c'est-à-dire à } I_{0} \times \int_{t_{0}}^{t_{0}+T} \mathrm{e}^{-t / \tau} \mathrm{d} t \text {. }
$$

L'intensité moyenne enregistrée par le photomultiplicateur est donc une fonction exponentielle de $t_{0}$.

Le CARY 14 étant étalonné en logarithmes décimaux, le déclin de fluorescence sera représenté par une droite $\log (I)=f\left(t_{0}\right)$ (voir fig. $3 b$ ). On a immédiatement la valeur de $n$ durées de vie, en soustrayant à une ordonnée quelconque $\operatorname{de} \log I$, la valeur $n \log e$.

Cas des grandes durées de vie. - Pour une durée de vie supérieure à la seconde, on peut obtenir direc-

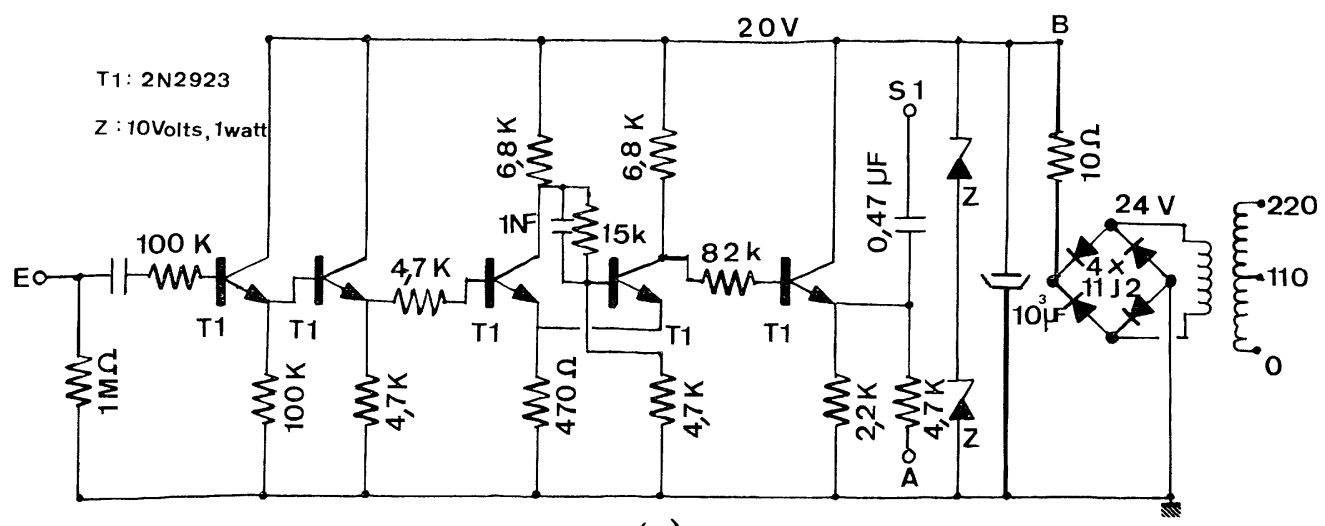

(a)

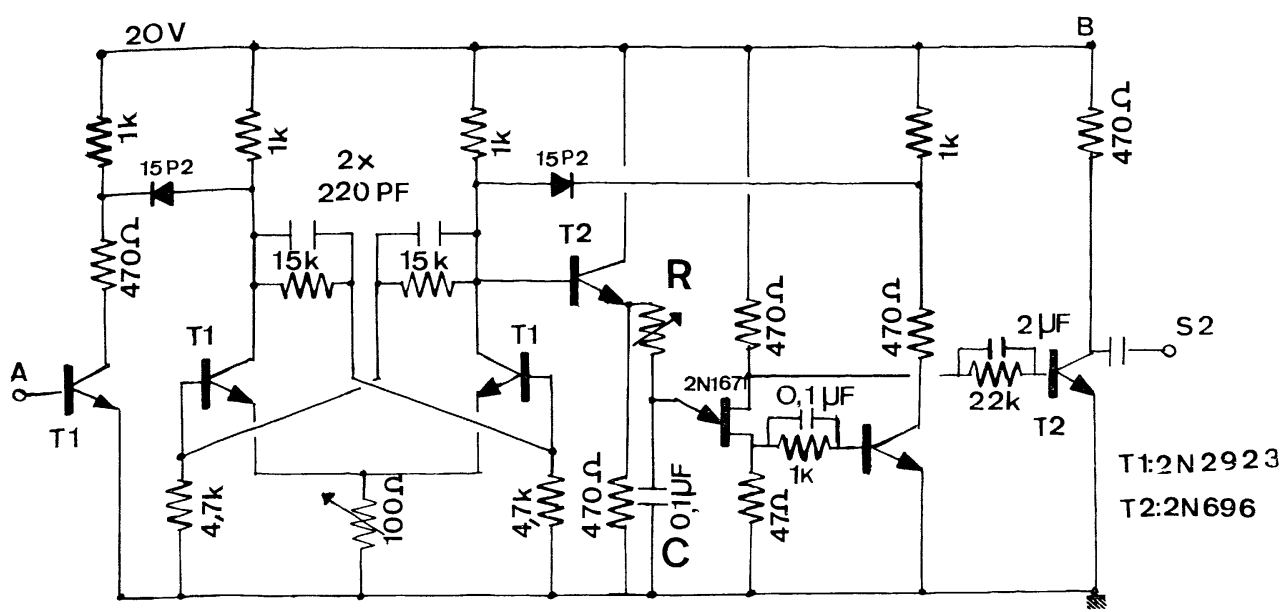

(b)

Fig. 4. - Schéma électronique du retardeur d'impulsion.

a) Sélecteur d'impulsion. - b) Bascule de retard. 
tement la trace linéaire du déclin sur l'enregistreur logarithmique du CARY. Le temps de réponse de la plume est négligeable dans ce cas et il suffit d'arrêter l'excitation à un moment donné. La décroissance peut ainsi être suivie depuis une intensité $I$ jusqu'à $I / 100$.
Retardateur d'impulsions (fig. 4). - Cet appareil comporte deux parties :

a) Le sélecteur D'impulsions. - Les circuits d'entrée à haute impédance servent à trier les impulsions de synchronisation du CARY en T 20. Ils se

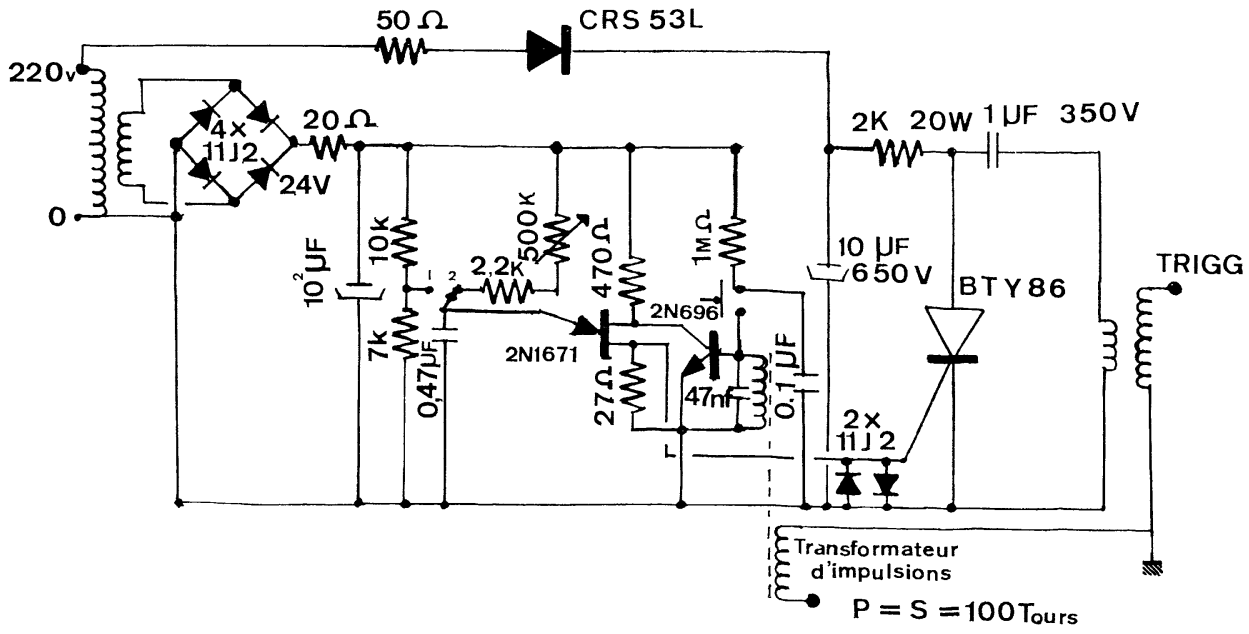

Fig. 5. - Schéma du déclencheur synchronisé.

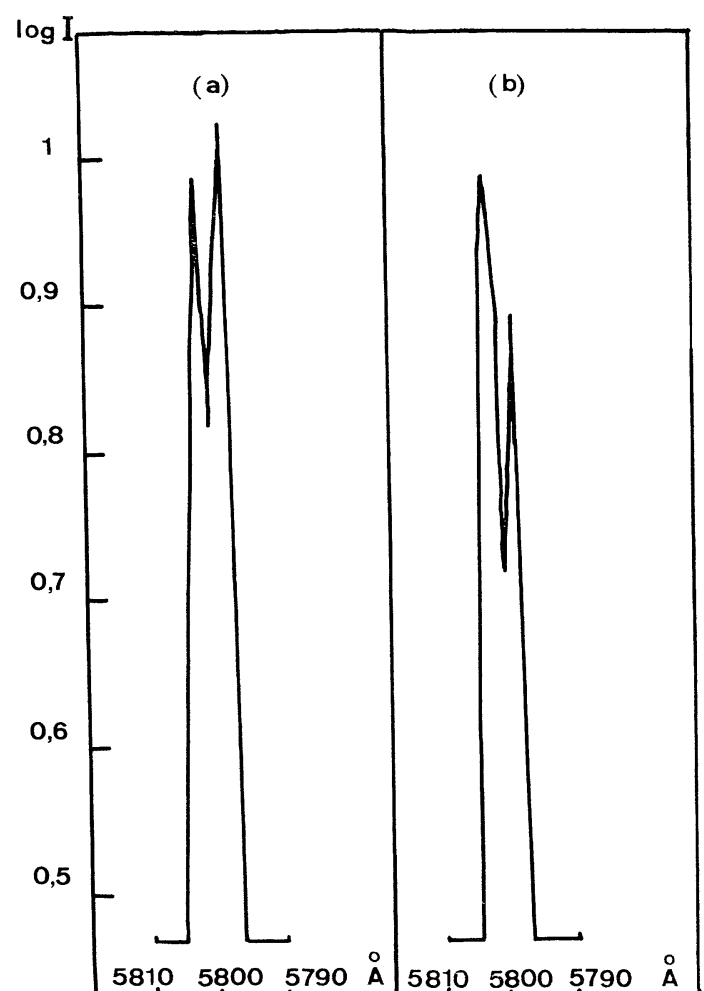

Fig. 6. - Transition ${ }^{5} D_{0} \rightarrow{ }^{7} F_{0}$ en fluorescence d'un mélange de tri- et tétra-benzoylacétonate d'europium en solution.

a) Observée avec un "temps d'observation" $t_{0}=0$.

b) Observée avec un "temps d'observation » $t_{0}=0,5 \mathrm{~ms}$. composent d'un étage abaisseur d'impédance suivi d'une bascule de Schmitt qui sélectionne les grands créneaux qui ont lieu toutes les 40 millisecondes (voir fig. 1b).

b) La bascule de retard. - C'est une bascule bistable bouclée avec un montage astable à unijonction ramenant celle-ci à l'état initial après un retard fixé par la constante de temps R. C. Pour la facilité d'utilisation, $R$ est constitué d'un commutateur à 12 positions (fluorescence - retard variable de 0 à $40 \mathrm{~ms}$ et 10 retards fixes correspondant à des temps d'observation variant de 1 à $35 \mathrm{~ms}$ ).

Déclencheur synchronisé (fig. 5). - Les impulsions HT de $15 \mathrm{kV}$ avec une fréquence de répétition de $25 \mathrm{~Hz}$ sont réalisées à l'aide d'un transformateur d'impulsion THT. La décharge d'un condensateur dans le primaire est synchronisée avec les impulsions sortant du retardeur par un thyratron au silicium, lui-même amorcé par un transistor unijonction.

Exemples d'utilisation. - Le dispositif réalisé est particulièrement adapté à l'étude des transitions spontanées intenses obtenues dans les cristaux. verres dopés et liquides organiques, des matériaux pour lasers solides. Les durées de vie de l'ordre de la milliseconde et la structure fine des raies de luminescence peuvent être mesurées et comparées avec précision.

Par exemple dans la luminescence du tétrabenzoylacétonate-sodium d'europium, la raie due à la transition ${ }^{5} D_{0} \rightarrow{ }^{7} F_{0}$ est double dans la solution alcoolique refroidie à $77^{\circ} \mathrm{K}$. Le CARY 14 permet 
de distinguer deux maxima distants de $3 \AA$. Or cette transition atomique ne peut théoriquement donner lieu à aucune décomposition en sousniveau [2]. Le luminographe réalisé permet de vérifier immédiatement que ces deux raies proviennent de niveaux différents, donc de composés distincts. En effet, la comparaison des spectres obtenus pendant l'excitation avec un temps d'observation de $0,5 \mathrm{~ms}$ ( fig. 6) montre clairement que les durées de vie des deux raies sont différentes. Ceci confirme donc la décomposition partielle du chélate à quatre ligandes en chélate à trois ligandes dans la solution. Pour ce dernier la raie correspondante ${ }^{5} D_{0} \rightarrow{ }^{7} F_{0}$ est décalée de $3 \AA$ et la durée de vie est effectivement plus courte que celle du quatre ligandes [3].

Les auteurs tiennent à remercier $\mathrm{H}$. Poncet et M. Boisleve pour leur collaboration dans la réalisation des montages.

Manuscrit reçu le 2 mai 1966.

\section{RÉFÉRENCES}

[1] Meyer et Astier, J. Physique, 1963, 24, 1089.

[2] Brecher, Samelson et Lempicki, J. Chem. Physics, 1965, 42, 1081
[3] Meyer, Poncet et Verron, C. R. Acad. Sc., 1964, 259, 103. 